\title{
Function Projective Lag Synchronization of Hyperchaotic Systems via Impulsive Control
}

\author{
Jingui Liu \\ Faculty of Mathematics and Physics, Huaiyin Institute of Technology \\ Huaian, Jiangsu, China, 223001
}

\begin{abstract}
In this paper, we consider function projective lag synchronization of two identical hyperchaotic systems. Impulsive control method is employed to design a controller to achieve function projective lag synchronization. Based on the Lyapunov stability theorem and impulsive control theory, a sufficient condition is given for the asymptotical stability of the error dynamics. Finally, numerical simulation is provided to show the effectiveness of the proposed controller design methodology.
\end{abstract}

Keywords-function projective lag synchronization; impulsive control; hyperchaotic systems

\section{INTRODUCTION}

During the last two decades, there has been considerable interest in the synchronization of chaotic(hyperchaotic) dynamic systems since Pecora and Carroll introduced a method to synchronize two identical chaotic systems with different initial conditions. Many different techniques have been proposed, such as variable structure control, OGY method, adaptive control, active control, DFC control, backsteppping technique, and so on. Recently, Li proposed a new synchronization scheme, called function projective synchronization, which extended the projective synchronization. The idea of function projective synchronization is to synchronize the states of the master and slave system up to a scaling function. Since it can provide more secure communication in application to secure communication, it has attracted attention of scientists and engineers.

In the practical engineering applications, time delay is inevitable. For instance, in the telephone communication system, the voice one hears on the receiver side at time $t$ is often the voice from the transmitter side at time $t-\gamma$. So, in many cases, it is more reasonable to require the slave system to synchronize the master system with a time delay $\gamma$. Therefore, motivated by the existing works and take into account of the time delay, a new kind of chaos synchronization is introduced in this paper, which is named as function projective lag synchronization(FPLS).

Impulsive control is a control paradigm based on impulsive differential equations. It has attracted the attention of many researchers for its accomplish easily, the lower cost and little wastage in recent years. The theory of impulsive differential equations and impulsive control have been intensively studied, see [1-7] and relevant references therein. To the best of our knowledge, there are few results in discussing the function projective lag synchronization via impulsive control.
Motivated by the above discussion, our main aim in this paper is to discuss FPLS of hyperchaotic systems. Based on the Lyapunov theory and impulsive control theory, an impulsive controller is designed to achieve FPLS.

\section{FORMULATION OF THE PROBLEM}

Consider the following master systems

$$
\dot{x}(t)=A x(t)+\varphi(x),
$$

where $x(t) \in R^{n}$ is the state vector of master systems. In order to achieve the function projective lag synchronization, we construct the impulsively controlled slave system as below

$$
\left\{\begin{array}{l}
\dot{y}(t)=A y(t)+\varphi(y), t \neq t_{k}, \\
\Delta y=B_{k}(\alpha(t) x(t-\gamma)-y(t)), t=t_{k}, k=1,2, \cdots \cdots
\end{array}\right.
$$

where $y(t) \in R^{n}$ is the state vector of slave systems, $\Delta y\left(t_{k}\right)=y\left(t_{k}^{+}\right)-y\left(t_{k}^{-}\right), y\left(t_{k}^{+}\right)=\lim _{t \rightarrow t_{k}^{+}} y(t), y\left(t_{k}^{-}\right)=\lim _{t \rightarrow t_{k}^{-}} y(t)$. In general, it is assumed that $y\left(t_{k}^{-}\right)=y\left(t_{k}\right)$, which means that $y(t)$ is continuous from the left. The impulsive instant sequence $t_{k}, k=1,2, \cdots \cdots$ satisfies $0<t_{1}<t_{2}<\cdots<t_{k}<t_{k+1}$ $<\cdots, t_{k} \rightarrow \infty$ as $k \rightarrow \infty$, and $B_{k}$ denote the impulsive control gains, $\alpha(t) \neq 0$ for all $t$ is the scaling function, the constant $\gamma$ denotes delay.

Let the error states $e(t)=\alpha(t) x(t-\gamma)-y(t)$. If, for any initial states $x_{0}$ and $y_{0}$, we have $e(t)=\alpha(t) x(t-\gamma)-y(t)$

$\rightarrow 0(t \rightarrow \infty)$, then these two systems (1) and (2) achieve FPLS. Thus the error dynamical systems can be described as

$$
\left\{\begin{array}{l}
\dot{e}(t)=A e(t)+\alpha(t) \varphi(x(t-\gamma))-\varphi(y)+\dot{\alpha}(t) x(t-\gamma), t \neq t_{k} \\
\Delta e(t)=-B_{k}(\alpha(t) x(t-\gamma)-y(t)), t=t_{k}, k=1,2, \cdots \cdots \\
e\left(t_{0}^{+}\right)=e_{0}
\end{array}\right.
$$


The purpose of this paper is to find some conditions on the control gains $B_{k}$ and the impulsive distances $t_{k+1}-t_{k}<\Delta<\infty$ ( $k=1,2, \cdots \cdots)$ for guaranteeing the FPLS of systems (1) and (2).

\section{FPLS OF HYPERCHAOTIC LORENZ SYSTEM}

In this section, we choose hyperchaotic Lorenz system as the master and slave system. The system is given as follows

$$
\dot{x}(t)=A x(t)+\varphi(x)
$$

where $x(t)=\left(x_{1}, x_{2}, x_{3}, x_{4}\right)^{T}$ is state vector, $a, b, c, k_{1}$ and $k_{2}$ are real parameters, and

$$
A=\left(\begin{array}{cccc}
-a & a & 0 & 0 \\
b & -1 & 0 & 0 \\
0 & 0 & c & 0 \\
k_{1} & k_{2} & 0 & 0
\end{array}\right), \varphi(x)=\left(\begin{array}{c}
0 \\
-x_{1} x_{3} \\
x_{1} x_{2} \\
0
\end{array}\right)
$$

We assume that the scaling function $\alpha(t)$ is a continuously differentiable bounded function, and $\|\dot{\alpha}(t)\| \leq \rho$, where $\rho$ is a constant. Then we obtain the following Theorem.

Theorem Let $\beta_{k}$ and $\mu$ be the largest eigenvalues of $\left(I-B_{k}\right)^{T}\left(I-B_{k}\right)$ and $\left(A^{T}+A\right) / 2$, respectively. If there exists a constant $\zeta>1$ such that the following condition holds

$$
\begin{aligned}
& \ln \left(\zeta \beta_{k}\right)+2\left[\mu+M+N_{1}\left(\sqrt{2} M+N_{2}+N_{3}\right) \varsigma+\rho \chi \varsigma\right]\left(t_{k}-t_{k-1}\right) \\
& \leq 0, k=1,2, \cdots \cdots .
\end{aligned}
$$

Then the trivial solution of error dynamical system (3) is asymptotically stable for $\varsigma\|e\| \geq 1$, where $\varsigma$ is positive, and $\|x\| \leq \chi,\|\dot{\alpha}(t)\| \leq \rho, M=\max \left\{\left|x_{1}\right|,\left|x_{2}\right|,\left|x_{3}\right|\right\},\left|y_{1}\right| \leq N_{1}$,

$$
\left|y_{2}\right| \leq N_{2},\left|y_{3}\right| \leq N_{3} \text {. }
$$

Proof Consider the Lyapunov function $V(t, e)=\frac{1}{2} e^{T}(t) e(t)$

When $t \neq t_{k}$, the derivative of $V(t, e)$ along the solution of (3) is

$$
\begin{aligned}
& D^{+} V(t, e)=\frac{1}{2}\left\{[A e(t)+\alpha(t) \varphi(x(t-\gamma))-\varphi(y)+\dot{\alpha}(t) x(t-\gamma)]^{T}\right. \\
& \left.e(t)+e^{T}(t)[A e(t)+\alpha(t) \varphi(x(t-\gamma))-\varphi(y)+\dot{\alpha}(t) x(t-\gamma)]\right\} \\
& =\frac{1}{2} e^{T}(t)\left[A^{T}+A\right] e(t)-x_{3}(t-\gamma) e_{1} e_{2}+x_{2}(t-\gamma) e_{1} e_{3}-y_{1} \\
& {\left[x_{3}(t-\gamma)-y_{3}\right]+e_{2} y_{1}\left[x_{2}(t-\gamma)-y_{2}\right] e_{3}+\dot{\alpha}(t) x^{T}(t-\gamma) e(t) .}
\end{aligned}
$$

for $\varsigma\|e\| \geq 1$, one can conclude that $\varsigma\|e\|^{2} \geq\|e\|$, and therefore

$$
\begin{aligned}
& D^{+} V(t, e) \leq \frac{1}{2} e^{T}(t)\left[A^{T}+A\right] e(t)+M\left|e_{1}\right|\left|e_{2}\right|+M\left|e_{1}\right|\left|e_{3}\right| \\
& \quad+N_{1} M\left(\left|e_{2}\right|+\left|e_{3}\right|\right)+N_{1} N_{3}\left|e_{2}\right|+N_{1} N_{2}\left|e_{3}\right|+2 \rho M\|e\| \\
& \leq \mu e^{T}(t) e(t)+M e^{T}(t) e(t)+\left[N_{1}\left(\sqrt{2} M+N_{2}+N_{3}\right)\right. \\
& +\rho \chi] \varsigma e^{T}(t) e(t) \\
& =2\left[\mu+M+N_{1}\left(\sqrt{2} M+N_{2}+N_{3}\right) \varsigma+\rho \chi \varsigma\right] V(t, e) .
\end{aligned}
$$

when $t=t_{k}$, we have

$$
\begin{gathered}
V\left(t_{k}, e-B_{k} e\right)=\frac{1}{2}\left[e\left(t_{k}\right)-B_{k} e\left(t_{k}\right)\right]^{T}\left[e\left(t_{k}\right)-B_{k} e\left(t_{k}\right)\right] \\
=\frac{1}{2} e^{T}\left(t_{k}\right)\left(I-B_{k}\right)^{T}\left(I-B_{k}\right) e\left(t_{k}\right) \\
\leq \frac{1}{2} \lambda_{\max }\left(\left(I-B_{k}\right)^{T}\left(I-B_{k}\right)\right) e^{T}\left(t_{k}\right) e\left(t_{k}\right) \\
=\beta_{k} V\left(e\left(t_{k}\right)\right) .
\end{gathered}
$$

Then, it follows from Theorem 1 in [2] that the origin of the system (3) is asymptotically stable. This completes the Proof.

\section{NUMERICAL SimUlation}

In order to verify and illustrate the effectiveness of the proposed method, we show the numerical simulation of the hyperchaotic Lorenz system (4). The system is hyperchaotic for the parameters $a=10, b=28, c=-8 / 3$, and $k_{1}=-9.3, k_{2}=1$ with initial value $x_{0}=(13.8,-14,13,13)$ as shown in Figure I.

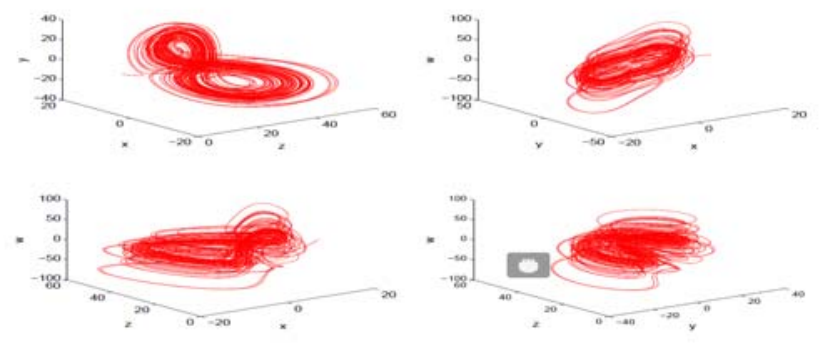

FIGURE I. THE HYPERCHAOTIC BEHAVIOR OF THE SYSTEM

By simple calculation, the eigenvalues of the matrix $\left(A^{T}+A\right) / 2$ are 14.3387,-25.7299,0.3913, and -2.6667 , we have $\mu=\lambda_{\max }\left(\left(A^{T}+A\right) / 2\right)=14.3387$. To test Theorem, we set $\gamma=0.018, \varsigma=1.05, \zeta=1.02$. The impulsive gain matrix $B_{k}$ is selected as a constant matrix $B=\operatorname{diag}\{\sigma, \sigma, \sigma, \sigma\}=\operatorname{diag}$ $\{0.8625,0.8625,0.8625,0.8625\}$. Then we can easily obtain 
$\beta_{k}=\beta=(1-\sigma)^{2}=0.0189$. Hence the estimation of the stable region is $0 \leq \Delta \leq 0.000211$.

We choose the impulsive interval $\Delta=0.0002$, which satisfies the conditions of the Theorem. In order to show the effect of control input, $u(t)$ is applied at 10 sec. Figure II shows the errors with scaling function $\alpha(t)=\sin 2 t+1.5$, and the dynamical behaviors of state variables are presented in Figure III. From Figure II and III, we can see that all the states variables of the hyperchaotic system achieve FPLS, which shows the effectiveness of our method via impulsive control.
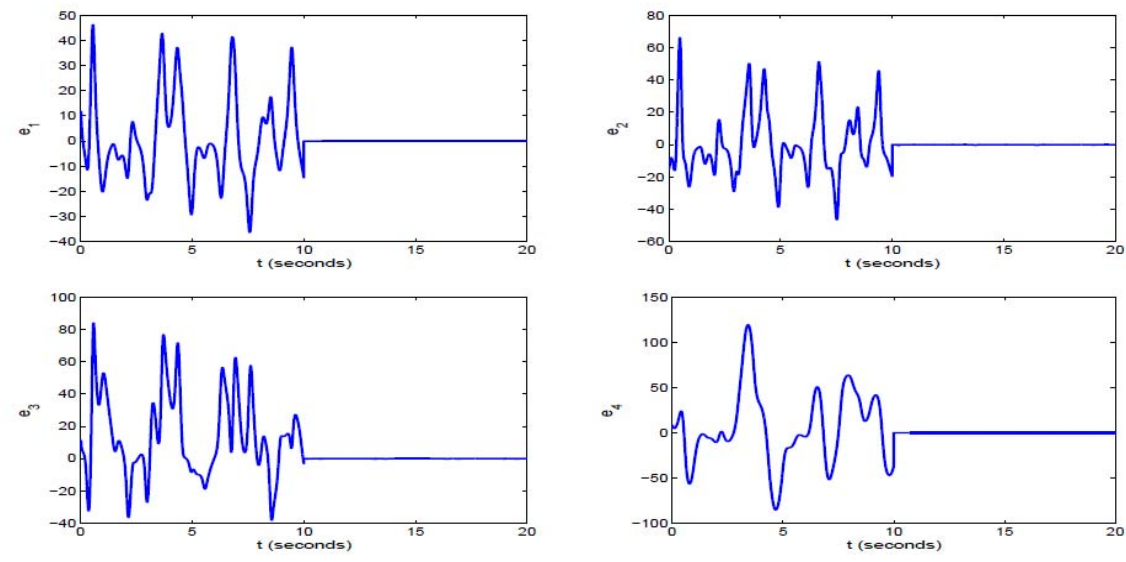

FIGURE II. SYNCHRONIZATION ERRORS OF THE SYSTEM
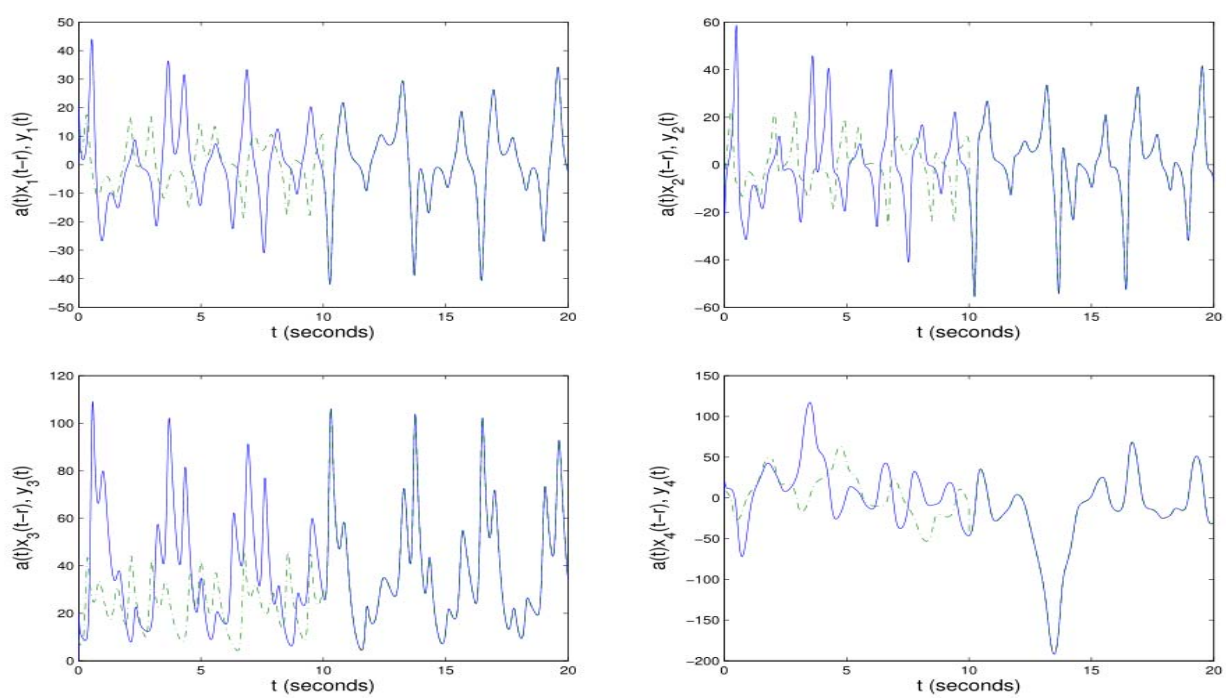

FIGURE III. DYNAMICAL BEHAVIORS OF STATE VARIABLES OF THE SYSTEM

\section{CONCLUSION}

This paper has investigated the FPLS problem via impulsive control. Stability condition is proposed and an impulsive controller is also designed. The effectiveness of the presented method has been illustrated by numerical simulation.

\section{REFERENCES}

[1] V. Laskshmikantham, D. D. Bainov, and P. S. Simeonov, Theory of Impulsive Differential Equations. Singapor, World Scientific, 1989.

[2] T. Yang, Impulsive Systems and Control: Theory and Application. New York, Nova Science, 2001.
[3] X. Z. Liu and G. Ballinger, "Uniform asymptotic stability of impulsive delay differential equations”, Comput Math Appl , vol. 41, pp. 903-915, 2001.

[4] X. H. Zhang, A. Khadra, D. Yang, and D. Li, "Unified impulsive fuzzymodel-based Controllers for Chaotic Systems with Parameter Uncertainties via LMI”, Commun Nonlinear Sci Numer Simulat, vol. 15, pp. 105-114, 2010

[5] G. M. Liu and W. Ding, "Impulsive synchronization for a chaotic system with channel time-delay”, Commun Nonlinear Sci Numer Simulat, vol. 16, pp. 958-965, 2011.

[6] Y. B. Yu, F. L. Zhang, Q. S. Zhong, X. F. Liao, and J. B. Yu, "Impulsive control of Lurie system”, Comput Math Appl, vol. 56, pp. 2806-2813, 2008. 
[7] X. Z. Liu, K. L. Teo, and Y. Zhang, “Absolute stability of impulsive control systems with time delay", Math Comput Model, vol. 62, pp. 429453, 2005. 